\title{
Single-Entity Electrochemistry for Digital Biosensing at Ultralow Concentrations
}

\author{
Serge G. Lemay* and Taghi Moazzenzade
}

Cite This: Anal. Chem. 2021, 93, 9023-9031

Read Online

ABSTRACT: Quantifying ultralow analyte concentrations is a continuing challenge in the analytical sciences in general and in electrochemistry in particular. Typical hurdles for affinity sensors at low concentrations include achieving sufficiently efficient mass transport of the analyte, dealing with slow reaction kinetics, and detecting a small transducer signal against a background signal that itself fluctuates slowly in time. Recent decades have seen the advent of methods capable of detecting single analytes ranging from the nanoscale to individual molecules, representing the ultimate mass sensitivity to

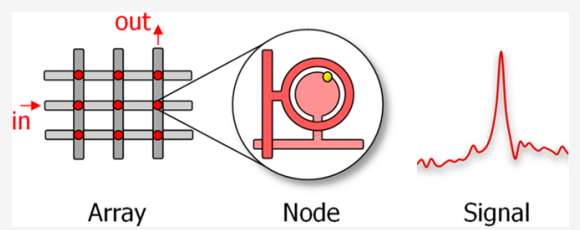
these analytes. However, single-entity detection does not automatically translate into a superior concentration sensitivity. This is largely because electrochemical transducers capable of such detection are themselves miniaturized, exacerbating mass transport and binding kinetic limitations. In this Perspective, we discuss how these challenges can be tackled through so-called digital sensing: large arrays of separately addressable single-entity detectors that provide real-time information on individual binding events. We discuss the advantages of this approach and the barriers to its implementation.

\section{INTRODUCTION}

A salient theme in analytical science in recent decades has been the miniaturization of detection systems. Driven by the nanotechnology revolution, an important end point of this process has been the development of methods capable of detecting and interrogating individual microscopic entities ranging from inorganic and biological nanoparticles to single molecules. These methodological developments have opened new windows into the microscopic world and enabled fundamental and exploratory studies at the molecular scale in practically all areas of the physical sciences.

Electrochemistry is no exception to this trend. Conventional methods such as voltammetry and amperometry are in principle straightforward to scale down, even to the nanoscale, by decreasing the size of the electrodes employed. Interestingly, the detection of individual target particles or molecules can become possible when the size of the working electrode(s) approaches that of the targets. This has led to the development of so-called single-entity electrochemistry (SEE), as recently expounded by Baker. ${ }^{1}$

The best-known example of a SEE analytical method is the classic Coulter counter, in which modulation of the ionic conduction through an aperture is utilized to count and size particles. ${ }^{2}$ This concept has been scaled down to molecular scale in both solid-state ${ }^{3,4}$ and biological ${ }^{5}$ nanopores, the latter having become so sophisticated as to permit the sequencing of nucleic acids. ${ }^{6-9}$ Other examples of nanoscale SEE approaches include nanoelectrode-based studies for single-cell studies, ${ }^{10}$ electrogeneration of surface nanobubbles, ${ }^{11}$ single-ion-channel patch clamp recordings, ${ }^{12,13}$ scanning-probe methods ranging from electrochemical scanning tunneling microscopy ${ }^{14,13}$ to scanning electrochemical cell microscopy (SECCM), ${ }^{16-18}$ single-molecule redox cycling, ${ }^{19,20}$ and various forms of particle-impact electrochemistry. ${ }^{21-24}$

Typical SEE signals have a different character from that of conventional electrochemical data. Because individual singleentity events occur at random intervals, data typically consist of a mixture of quiescent periods in which very little happens, punctuated by sudden jumps or spikes in the signal corresponding to the detection of the analyte. This phenomenology is illustrated in Figure 1 for various amperometry-based methods. Determining a concentration from these signals requires counting events in time rather than measuring an average response.

Single-entity methods have however made relatively few inroads in the sensing of ultralow analyte concentrations. This may at first appear surprising: does not single-molecule detection represent the ultimate sensitivity? Indeed, the ability to detect a single analyte particle or molecule represents remarkable mass sensitivity for that analyte. One cannot detect fewer than one elementary entity. In practice, however, the goal is typically to determine the concentration of an analyte, and concentration sensitivity represents the key figure of merit. A high mass sensitivity does not necessarily translate into a high concentration sensitivity. The very characteristics lending a

Received: February 3, 2021

Accepted: June 9, 2021

Published: June 25, 2021 

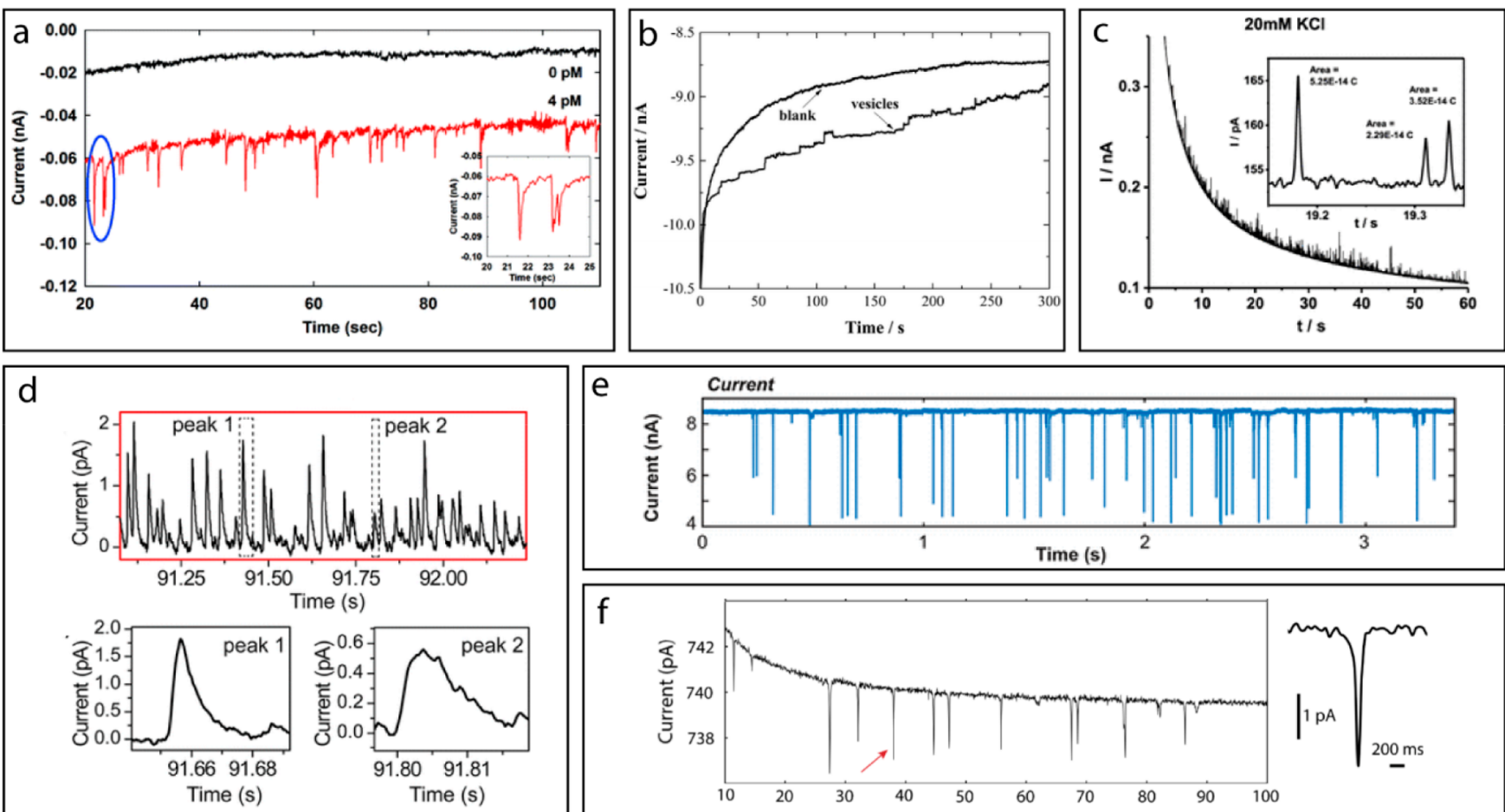

$f$

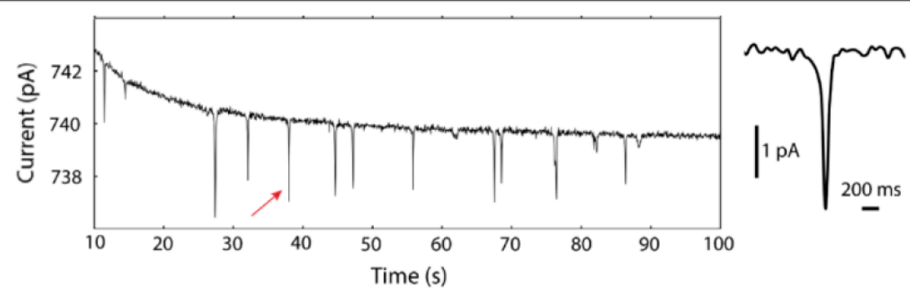

Figure 1. Examples of discrete signals in amperometry-based SEE. (a) Mediated faradaic impact: current spikes occur due to electrocatalytic water oxidation upon impact of $\mathrm{IrO}_{x}$ nanoparticles on a $\mathrm{NaBH}_{4}$-treated Pt UME. ${ }^{33}$ Adapted with permission from ref 33 . Copyright 2010 American Chemical Society. (b) Current blockade impact: steps in the anodic current from oxidation of the $\mathrm{Fe}(\mathrm{CN})_{6}{ }^{4-}$ mediator are observed upon impact of vesicles on a Pt ultramicroelectrode. ${ }^{34}$ Adapted with permission from ref 34. Copyright 2015 American Chemical Society. (c) Direct faradaic impact: Ag nanoparticles are oxidized upon making contact with an electrode, leading to current spikes. ${ }^{35}$ Adapted with permission from ref 35. Copyright 2019 American Chemical Society. (d) Scanning electrochemical cell microscopy (SECCM): current spikes are observed when Au nanoparticles are immobilized upon an alkanethiol-modified Au electrode. ${ }^{36}$ Adapted with permission from ref 36 . Copyright 2015 American Chemical Society. (e) Solid-state nanopore: transient dips occur in the ionic current through the (plasmonic) nanopore upon translocation of single DNA molecules. ${ }^{37}$ Adapted with permission from ref 37. Copyright 2018 American Chemical Society. (f) Electroosmotically driven current blockade: current from oxidation of the ferrocene dimethanol mediator dips during convectively driven transient passage of polystyrene beads over a Pt ultramicroelectrode. ${ }^{38}$ Adapted with permission from ref 38. Copyright 2020 American Chemical Society.

high mass sensitivity to a transducer can even explicitly preclude a high concentration sensitivity.

We argue that single-entity electrochemical detectors are intrinsically handicapped in measuring low concentrations due to their small physical dimensions. These limitations can however be circumvented by employing large arrays of independently addressable transducers. Consistent with recent usage in both SEE and single-molecule optical measurements, ${ }^{25-28}$ we employ the term "digital sensing" to refer to this combination of single-entity, real-time, and parallelized detection. This terminology evokes the high degree of integration characteristic of modern electronics. It also reflects the discrete nature of the output signals, which in an abstract sense consist of a stream of discrete events as a function of time rather than an averaged sensor response. This represents a more stringent use of the term compared to digital microfluidics $^{29,30}$ or digital ELISA, ${ }^{31,32}$ where discrete events are usually detected via chemical postamplification.

More precisely, we define a digital sensor as an ensemble of independent nodes, each consisting of a device capable of single-entity detection. In practice this corresponds to generating a (usually sudden) time-resolved signature each time that a microscopic event of interest takes place. Photomultiplier tubes and Geiger counters are classic examples that generate a burst of charge when a single photon or ionizing radiation particle impinges upon the detector. For an electrochemical sensor, each node consists of a separate SEE experiment that can be independently sampled and analyzed. In its simplest form, each feature in the node signal (such as the steps and pulses in Figure 1) can be assigned one-to-one to the occurrence of an event of interest (the binding of an analyte to a receptor, for example). More generally, the amplitude, duration and shape of single SEE events can be further analyzed to enhance specificity, as done for example in the analysis of nanopore signals. ${ }^{6-9}$ Such fingerprinting can be employed to selectively ignore false positives from interfering species, suppress instrumental noise, and eliminate baseline drift. Somewhat abstractly, the final output of the sensor as a whole can be considered to be the aggregate events from all of the nodes. In this sense, the output consists of a sequence of times at which each event took place together with the nature of that event, from which analyte concentrations are inferred. Translating the raw signals from the individual nodes into event counts and combining this information into a total sensor output can be carried out as postexperimental analysis, although performing parts of it in real time can help mitigate the sheer volume of data.

It is not necessarily a priori obvious that digital sensing can deliver performance superior to that of conventional approaches, which are based on large detectors integrating 
over a large number of microscopic events. Here, we first summarize fundamental challenges inherent to detecting ultralow concentrations. We pay particular attention to the main limitations of single-entity methods and address how parallelization can help lift some of the main obstacles. We then discuss the properties of the signals resulting from digital sensing. Finally, we argue that modern electronics are poised to provide the ideal platform for realizing digital sensors, noting that this may involve a shift from popular amperometry-based SEE approaches to high-frequency methods. While our focus is on electrochemical sensors, many of the general concepts that we consider here apply equally well to miniaturized optical and mechanical transducers.

\section{THE NEED FOR PARALLELIZATION}

SEE methods typically rely on electrodes with critical dimensions of a size that is not much larger than the size of the target itself. Simply put, it is easier to detect a small change in an electrical signal when it is not superimposed on a much larger background signal and the associated noise. However, decreasing the size of the working electrode comes at a price as it can limit the ultimate achievable performance of a sensor in several ways. In this section, we focus on the mechanisms by which minimization impacts theoretical detection limits.

First, decreasing the electrode size hinders mass transport of the analyte. Targets need to reach the sensor detection domain in order to be detected; the smaller the sensor, the lower the likelihood that a target will find the sensor in a given time interval. For nanoscale sensors, the time needed for analyte molecules to come into contact can become prohibitively large. This is best illustrated with a simple numerical example, as emphasized by Sheehan and Whitman. ${ }^{39}$ The steady-state diffusive flux (in analytes/second), $k_{\text {diff, }}$ to a miniaturized detector with characteristic lateral size $d$ has the general form $^{40,41}$

$$
k_{\text {diff }}=10^{3} \times D N_{\mathrm{A}} C \gamma d
$$

Here, $D$ is the diffusion coefficient of the analyte, $N_{\mathrm{A}}$ is Avogadro's number, $C$ is the molar concentration of the analyte, $d$ is the characteristic size of the detector, and $\gamma$ is a geometry-dependent factor of order unity (for example, $\gamma=2$ for a shrouded disk of diameter $d$ ). The total flux is proportional to $d$ and thus increases with increasing electrode size. Eq 1 is valid in the steady state, which is established after a brief transient with a duration comparable to the diffusion time, $d^{2} / 4 D .^{42}$ In practice, for miniaturized sensors, this transient is too short to be accessed, and eq 1 holds at essentially all times.

For small macromolecules $\left(D \approx 10^{-6} \mathrm{~cm}^{2} / \mathrm{s}\right)$ at a low concentration of $C=1 \mathrm{pM}$ diffusing to a nanoscale disk sensor $(d=100 \mathrm{~nm})$, steady state is achieved on the time scale of 25 $\mu$ s. The probability of even a single analyte reaching the electrode during this time is $\ll 1$. The steady-state flux then settles to a constant value of $\sim 0.01$ analytes/s. That is, one needs to wait on average $\sim 100 \mathrm{~s}$ between the moments when a single analyte arrives at the detector. Lower concentrations or larger analytes with lower diffusion coefficients demand correspondingly longer times, placing increasing limitations on the average number of detection events expected for a given electrode size and measurement time.

The above observations may at first appear to contradict the rule of thumb that "mass transport is more effective at miniaturized electrodes." It is true that, based on eq 1 , the analyte flux density scales as $d^{-1}$ and thus increases upon decreasing the electrode size. But, when working at the singleanalyte limit, what matters is the time needed for each analyte to reach the detector. This is determined by the total flux, not the flux density. Hence, the measurement time increases with smaller detector size.

A second complication introduced by sensor miniaturization stems from the properties of chemical equilibria and kinetics. These can place additional-and often more severelimitations on concentration sensitivity. As an illustration, consider Langmuir kinetics ${ }^{43}$ between an analyte at concentration $C$ and a single receptor, as described by the equation

$$
p=\frac{C}{K_{\mathrm{D}}+C}\left(1-\mathrm{e}^{-t / \tau}\right)
$$

Here, $p$ is the probability of the receptor being occupied by an analyte at time $t$ (starting from $p=0$ at $t=0$ ), $K_{\mathrm{D}}$ is the dissociation constant, and $\tau$ is the time constant for the reaction. In terms of the association and dissociation rates, $k_{\mathrm{a}}$ (units $\mathrm{M}^{-1} \mathrm{~s}^{-1}$ ) and $k_{\mathrm{d}}$ (units $\mathrm{s}^{-1}$ ), respectively, the model parameters take the form $K_{\mathrm{D}}=k_{\mathrm{d}} / k_{\mathrm{a}}$ and $\tau=\left(k_{\mathrm{a}} \mathrm{C}+k_{\mathrm{d}}\right)^{-1}$.

Eq 2 indicates that at low concentration $\left(C \ll K_{\mathrm{D}}\right)$ and in equilibrium $(t \rightarrow \infty)$ the probability of the receptor being occupied, $p \approx C / K_{\mathrm{D}}$, is much smaller than unity. That is, a receptor is most likely to remain unoccupied even at equilibrium. For a device incorporating a large number of receptors, $N_{\text {rec }}$ the average number of occupied receptors is $\left\langle N_{\text {occ }}\right\rangle=p N_{\text {rec }}$. In order to have at least one receptor occupied on average $\left(\left\langle N_{\text {occ }}\right\rangle=1\right)$, the minimum number of receptors is then $N_{\text {rec }}=1 / p=K_{\mathrm{D}} / C \gg 1$. If the detector is too small to accommodate this number of receptors, then $\left\langle N_{\text {occ }}\right\rangle<1$, and the sensor ceases to function in a conventional sense. In principle, it is still possible to determine $\left\langle N_{\text {occ }}\right\rangle$ when $\left\langle N_{\text {occ }}\right\rangle<$ 1 by measuring a time average over multiple association and dissociation cycles, but this usually requires an impractically long time.

Furthermore, the minimum requirement $\left\langle N_{\text {occ }}\right\rangle \geq 1$ is highly optimistic. Analyte binding is a stochastic process taking place at independent receptors, and random fluctuations occur around the average occupancy $\left\langle N_{\text {occ }}\right\rangle$. According to the binomial distribution, the root-mean-square fluctuation in the instantaneous value of the occupancy, $N_{\text {occ }}$ is $\Delta N=\sqrt{\left\langle N_{\text {occ }}\right\rangle}$ when $p \ll 1$. In order to use a measurement of $N_{\text {occ }}$ to deduce the analyte concentration $C$, it is therefore necessary to increase $\left\langle N_{\text {occ }}\right\rangle$ to a point where the relative error $\Delta N_{\text {occ }}$ / $\left\langle N_{\text {occ }}\right\rangle$ is sufficiently small. For example, based on the above, a $10 \%$ and $1 \%$ accuracy in the average occupancy $\left\langle N_{\text {occ }}\right\rangle$ requires $N_{\text {rec }}=10^{2} / p$ and $10^{4} / p$, respectively. Fitting these additional receptors onto the sensor increases its minimum size by a significant factor.

Finally, we have so far assumed that the receptors are in equilibrium with solution. Reaching equilibrium requires incubating the sample for a period equal to several times the relaxation time $\tau$. In the limit where $C \ll K_{\mathrm{D}}$, the expression for $\tau$ reduces to $\tau \approx k_{\mathrm{d}}^{-1}$. For molecules with a low dissociation rate, $\tau$ can thus prove prohibitively long (up to days). Alternatively, one does not wait for equilibrium and makes use of eq 2 to extract a concentration from the transient response. For short measurement times $(t \ll \tau)$ and low concentrations, eq 2 reduces to $p \approx\left(C / K_{\mathrm{D}}\right)(t / \tau)$. This occupancy is a factor of $t / \tau \ll 1$ smaller than the equilibrium result. Consequently, for 
a given desired average occupancy $\left\langle N_{\text {occ }}\right\rangle$, the required number of receptors further increases by a factor $\tau / t \gg 1$.

Summarizing, low equilibrium occupancy and long equilibration times combine to place rather stringent limitations on the minimum size of a sensor. For example, substituting typical numbers for short DNA at a concentration of $1 \mathrm{pM}, 10 \%$ accuracy, and $100 \mathrm{~s}$ measurement time yields $N_{\text {rec }} \geq 10^{2}\left(K_{\mathrm{D}} /\right.$ C) $(\tau / t) \approx 2 \times 10^{7}$. This estimate is based on representative values $^{44}$ of $K_{\mathrm{D}}=0.2 \mathrm{nM}$ and $\tau=10^{5} \mathrm{~s}$. Although specific values quoted in the literature vary substantially based on the specifics of different experiments, the conclusion that $N_{\text {rec }}$ is a very large number holds generally. At optimal packing, these receptors occupy $\sim 40 \mathrm{~nm}^{2} /$ receptor or $\sim 1000 \mu \mathrm{m}^{2}$ in total, corresponding to the area of a $\sim 36 \mu \mathrm{m}$ diameter disk electrode. This footprint is much larger than the typical nanoscale dimensions of devices capable of single-entity detection. Conversely, detecting a mere 100 target molecules at such a large electrode is at present essentially impossible by purely electrochemical means.

While here we have focused solely on diffusive mass transport and first-order kinetics using order-of-magnitude estimates, these illustrate a more general pattern. On the time scales deemed desirable for point-of-need assays, the number of single-entity events expected for a nanoscale sensor easily becomes far smaller than unity, let alone large enough to provide sufficient statistics for an accurate measurement. While performance may be somewhat improved using convection, this is usually insufficient to significantly alter the picture sketched above. For a more extensive and quantitative discussion, we refer the reader to the excellent review by Squires et al. ${ }^{45}$

These considerations lead to the conclusion that individual SEE detectors are largely unsuitable for detection at ultralow analyte concentrations, while devices large enough to satisfy the mass transport and binding kinetics requirements are too large to serve as SEE detectors. The same reasoning however suggests that the main limitations can be overcome through the massive parallelization of SEE detectors. This is most readily achieved through pixelation: replacing the single large electrode with a dense array of many individually addressable small electrodes, or nodes, each capable of SEE detection.

Consider an array of $N_{\text {node }}$ nodes arranged in the form of a disk, each consisting of a shrouded disk electrode of diameter $d$ supporting $N_{\text {rec }}$ receptors and separated by an average distance $\Delta$. Trivially, the total number of receptors in the array is increased by a factor of $N_{\text {node }}$ compared to a single node. Mass transport is also substantially increased. When $N_{\text {node }} \ll(\Delta / d$ $(-1)^{4}$, each electrode behaves independently and obeys eq $1 .^{46}$ In this case, the total rate of mass transport is also boosted by a factor of $N_{\text {node }}$. In the opposite limit where the number of nodes becomes large or the spacing between electrodes becomes small such that $N_{\text {node }} \gg(\Delta / d-1)^{4}$, mass transport becomes limited by hemispherical diffusion from the far field to the array as a whole, and $k_{\text {diff }}$ increases by a large yet more modest factor $\Delta \sqrt{N_{\text {node }}} / d{ }^{46}$ Interestingly, however, convective mass transport can be much more effectively employed in the latter case due to the larger Peclet numbers associated with the diameter of the array compared to a single electrode. ${ }^{47}$

For illustration, consider again the numerical example above where $2 \times 10^{7}$ DNA receptors are required. For $d=100 \mathrm{~nm}$ electrodes, this corresponds to $\sim 10^{5}$ electrodes. If these are positioned $1 \mu \mathrm{m}$ apart, the array has a diameter of $360 \mu \mathrm{m}$.
These parameters satisfy $N_{\text {node }} \gg(\Delta / d-1)^{4}=6600$, hence the diffusion-limited flux is essentially the same as that of a 360 $\mu \mathrm{m}$ electrode, yielding $k_{\text {diff }} \approx 40$ analyte $/ \mathrm{s}^{-1}$. This is sufficient to accommodate the maximum binding rate $k_{\text {ideal }}=$ $N_{\text {node }} N_{\text {rec }} k_{\mathrm{a}} C=1$ analyte/s.

This example demonstrates how an electrode array can allow simultaneously reconciling the demands of mass transport and chemical kinetics. More generally, the size and number of nodes, the geometry of the array and of the nodes, and the spacing between nodes can be concomitantly optimized using theoretically derived mass transport equations ${ }^{46}$ or numerical simulations. This optimization process will necessarily be subject to constraints imposed by the nature of the SEE measurement, the contents of a node, and the technology employed to implement the array.

\section{BENEFITS OF DIGITAL DETECTION}

Here, we discuss qualitatively the potential gains made by employing SEE detection methods.

Insensitivity to Baseline Drift. An important requirement for detecting a low concentration is to be able to detect a response above the background or baseline signal. Background may result for example from parasitic electrochemical reactions or small offsets in the measurement electronics. If the background signal was perfectly stable, the ability to measure small changes in detection signal would be limited only by the resolution of the readout electronics. In practice, however, the baseline signal fluctuates due to, e.g., temperature variations, electrode aging, and the ubiquitous $1 / f$ noise characteristic of nonequilibrium processes. This renders the smallest signals occurring over long time scales, such as those that occur in affinity sensors, essentially impossible to distinguish from baseline drift.

A SEE detector, on the other hand, is often relatively insensitive to baseline drift. This is because there exists a clear separation of time scales between abrupt SEE events and the (usually much slower) baseline fluctuations. Intuitively, it is clear that steps and spikes that occur on a subsecond time scale are easily identified against a smoothly varying background signal that evolves on the scale of minutes or longer. This remains true even when the amplitude of the discrete events is smaller than the amplitude of the slow background fluctuations.

Insensitivity to Electrode Fouling. In electrochemical methods, it is common for the surface of a working electrode to become fouled after prolonged exposure to a sample. In a conventional sensor, the corresponding change in sensitivity can lead to error. In digital sensing, slow changes in the response of individual nodes to discrete events do not a priori prevent identification. As long as the individual event signatures can be recognized, they can be counted and analyzed independently of their exact shape. This additional degree of robustness is especially relevant in analyses requiring long measurement times.

Accuracy. When considering the features of a digital sensor, it is crucial to make a distinction between the properties of the individual nodes and those of the sensor as a whole. Ideally, digital sensing would allow detecting every binding event and would exhibit no false positives from interfering species. In this idealized case, the sensor could exhibit near-perfect accuracy (vide infra). Importantly, however, this would not require that the individual node signals (e.g., the current in an amperometric measurement) 
exhibit perfect accuracy. Since the role of the node signals is to allow the identification and counting of events, it is sufficient that the characteristic signature of an event can be reliably recognized. Offsets or distortion of the signal are tolerable, insofar as they are taken into consideration so as not to preclude event detection and identification. The absolute accuracy of the raw output signal of the node thus becomes largely irrelevant in determining the accuracy of the sensor as a whole. This reduces the demands on the node instrumentation required to achieve a high sensor accuracy.

Advanced Signal Processing. There has been considerable effort devoted to applying modern statistical methods to the analysis of single-entity measurements. As a primary example, so-called hidden Markov model descriptions ${ }^{48,49}$ permit the construction of sophisticated models of microscopic dynamics from complex time-resolved measurements even with limited a priori knowledge. These approaches have been extensively applied for example to the interpretation of singleion-channel recordings ${ }^{50}$ and Förster resonance energy transfer (FRET) measurements. ${ }^{51,52}$ While so far underutilized in most SEE experiments, these methods can be harnessed in the future for interpreting signals in digital SEE sensing, with expected boosts in both sensitivity and selectivity.

\section{ACCURACY, PRECISION, SENSITIVITY, AND DYNAMIC RANGE}

In order to paint a more quantitative picture of the noise properties of digital sensors, we consider some performance indicators within the context of the simple Langmuir kinetics model introduced above. We focus on an affinity sensor at low occupancy $(p \ll 1)$. We ignore mass transport limitations and analyte unbinding, which corresponds to short times $(t \ll \tau \approx$ $\left.k_{\mathrm{d}}^{-1}\right)$. Under these assumptions, the analyte binding rate for one receptor is then given by $k_{\mathrm{a}} C$, and the total binding rate is $k_{\text {ideal }}$ $=N_{\text {node }} N_{\text {rec }} k_{\mathrm{a}} C$. The response of the sensor consists of a series of random events, or counts, as a function of time. This is illustrated in Figure $2 \mathrm{a}$ for two values of $k_{\text {ideal }}$ using numerically generated data. Each trajectory has a random character that nonetheless reflects an average rate $k_{\text {ideal }}$. The time required to achieve a certain number of counts is different for each trajectory, but it fluctuates around a mean value such that the average response remains predictable. This is illustrated in Figure $2 \mathrm{~b}$ and $\mathrm{c}$, which show histograms of the distribution of times required to reach a fixed number of events, $N_{\text {event }}=20$. These values cluster around a mean value but with a significant spread.

To represent imperfections in the nodes' ability to detect single events, we assume here for the average number of binding events from all the nodes in a time interval $\Delta t$, $\left\langle N_{\text {event }}\right\rangle$, the nonideal form

$$
\left\langle N_{\text {event }}\right\rangle=k_{\text {nonideal }} \Delta t=N_{\text {node }}\left(N_{\text {red }} \beta k_{\mathrm{a}} C+k_{\text {false }}\right) \Delta t
$$

Here, we introduced two parameters, $\beta$ and $k_{\text {false, to }}$ represent nonidealities. $\beta$ represents the probability that a binding event will actually be detected: $\beta=1$ represents a perfect detector, $0<\beta<1$ means that the sensor fails to detect some events, and $\beta>1$ corresponds to a situation in which a single target can trigger multiple counts. $k_{\text {false }}$ represents a rate of false events that cannot be distinguished from actual events; these could be the result of noise being mistakenly identified as an event or unwanted events caused by interfering species.

Note that this model is highly simplified for the sake of readability. For example, there are scenarios for which $k_{\text {false }}$
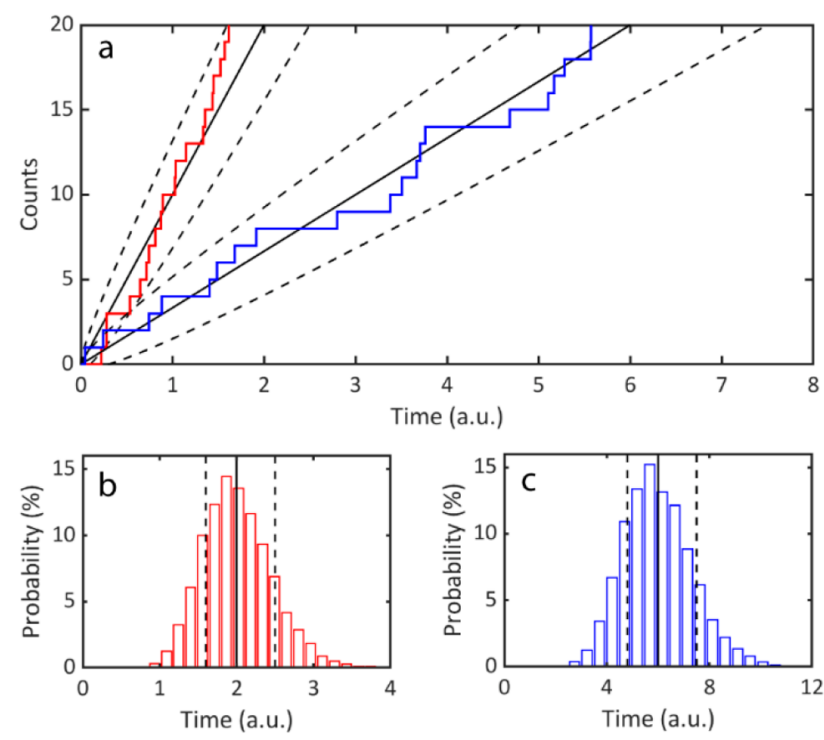

Figure 2. Simulated response of an ideal digital sensor. The sensor is described by a Poisson process with rate $k_{\text {ideal }}$. (a) Typical individual trajectories for $k_{\text {ideal }}=k_{0}($ red $)$ and $k_{\text {ideal }}=k_{0 / 3}(\mathrm{blue})$, where $k_{0}$ is an arbitrary rate constant. The solid black lines represent the average number of events at each time, $\left\langle N_{\text {event }}\right\rangle$, as predicted by eq 3 with $k_{\text {false }}$ $=0$. The dashed lines represent one standard deviation in $N_{\text {event }}$ as predicted by Poisson statistics. The trajectories were generated numerically via a simulated Poisson process. ${ }^{53}$ (b,c) Histograms showing the distribution of the time needed to generate 20 events for $k_{\text {ideal }}=k_{0}(\mathrm{~b}$, red $)$ and $k_{\text {ideal }}=k_{0} / 3\left(\mathrm{c}\right.$, blue) based on $10^{4}$ trajectories. Apart from a factor of 3 stretching in time in c compared to $b$ and small statistical fluctuations, the distributions are equivalent. This scaling property reflects eq 5 , which indicates that the relative error only depends on the number of counts.

could depend on either $N_{\text {rec }}$ or $C$, and the expressions below would need to be adapted for those cases.

Eq 3 can be inverted to yield an expression for the analyte concentration

$$
C=\frac{1}{N_{\text {red }} \beta k_{\mathrm{a}}}\left(\frac{N_{\text {event }}}{N_{\text {node }} \Delta t}-k_{\text {false }}\right)
$$

Determining the concentration in this way assumes that $k_{\text {false }}$ and the product $N_{\text {rec }} \beta k_{\mathrm{a}}$ are known a priori. Determining these parameters would entail a calibration process that would itself be subject to statistical error. For simplicity, we assume below that this error is sufficiently small as to be negligible in comparison to the statistical fluctuations arising during sensor operation.

During a measurement, the average $\left\langle N_{\text {event }}\right\rangle$ is not known; one performs a measurement to obtain a measured value $N_{\text {event }}$ which is subject to Poisson statistics. The probability of a false negative result $\left(N_{\text {event }}=0\right.$ even though $\left.\left\langle N_{\text {event }}\right\rangle \neq 0\right)$ is thus $\mathrm{e}^{-\left\langle N_{\text {event }}\right\rangle}$, which quickly becomes negligible as $\left\langle N_{\text {event }}\right\rangle$ increases. More generally, the standard deviation for $N_{\text {event }}$ is $\sqrt{\left\langle N_{\text {event }}\right\rangle}$, again from Poisson statistics (while the true value of $\left\langle N_{\text {event }}\right\rangle$ is not known, it can be estimated as the measured value, $N_{\text {event }}$, so long as $N_{\text {event }} \gg 1$ ). The corresponding standard deviation is given by (Supporting Information):

$$
\frac{\Delta C}{C}=\frac{1}{\sqrt{\left\langle N_{\text {event }}\right\rangle}} \frac{\left(1+N_{\text {node }} k_{\text {false }} \Delta t / N_{\text {event }}\right)^{1 / 2}}{1-N_{\text {node }} k_{\text {false }} \Delta t / N_{\text {event }}}
$$


Accuracy and Precision. In the absence of false positive events $\left(k_{\text {false }}=0\right)$, eq 4 indicates that the accuracy of the measurement depends on the degree to which the multiparameter product $\beta N_{\text {rec }} k_{\mathrm{a}}$ is known. The accuracy is thus set by the uncertainty in $k_{\mathrm{a}}$ (a molecular property), $N_{\text {rec }}$ (which depends on the reliability and reproducibility of electrode functionalization), and $\beta$ (which may depend on both receptor immobilization and the ability to recognize single events in the node signals).

Again for $k_{\text {false }}=0$, eq 5 indicates that the relative precision, $\Delta C / C$, depends only on $\left\langle N_{\text {event }}\right\rangle$, independently of the value of the physical parameters of the system. $\Delta C / C$ decreases monotonically with increasing $\left\langle N_{\text {event }}\right\rangle$, indicating that, not surprisingly, the precision improves with increased measurement time $\Delta t$. This also indicates that the same desired degree of precision can be attained for measurements at different analyte concentrations by letting $\Delta t$ vary for each measurement such that a desired value for $N_{\text {event }}$ (and thus $\Delta C / C$ ) is achieved. ${ }^{27}$ This property is reflected in Figure $2 \mathrm{~b}$ and $\mathrm{c}$, where the distributions of times for achieving $N_{\text {event }}=20$ are seen to be independent of $k_{\text {ideal }}$ apart from an overall scaling factor. In practice, taking advantage of this property would require realtime event recognition.

In cases where false positives occur $\left(k_{\text {false }}>0\right)$, the situation is essentially unchanged so long as the number of false positives, $N_{\text {node }} k_{\text {false }} \Delta t$, remains much smaller than the total number of measured events, $N_{\text {event }}$. When the majority of events becomes false events, on the other hand, eq 5 indicates that the relative error increases significantly. Reducing the rate of false events thus becomes critical for improving precision at the lowest achievable concentrations.

Sensitivity. As per the IUPAC definition, the sensitivity is the slope of the response curve. ${ }^{54}$ Eq 3 indicates that the response is linear in $C$ (as opposed to, e.g., logarithmic), yielding a constant sensitivity

$$
\text { Sensitivity }=\frac{d\left\langle N_{\text {event }}\right\rangle}{d C}=N_{\text {node }} N_{\text {red }} \beta k_{\mathrm{a}} \Delta t
$$

The sensitivity is proportional to $N_{\text {node }}$ independently of the values of the node parameters.

Dynamic Range. The measurement must be achievable in a reasonable time. Combining eq 3 and eq 5 yields for the minimum measurement time at a desired relative precision (taking $k_{\text {false }}=0$ for the moment)

$$
\Delta t=\frac{1}{N_{\text {node }} N_{\text {red }} \beta k_{\mathrm{a}} C(\Delta C / C)^{2}}
$$

This minimum time can in principle be made arbitrarily small by increasing $N_{\text {node }}$. On the other hand, the precision deteriorates once $C \lesssim k_{\text {false }} / N_{\text {rec }} \beta k_{a}$. This depends only on the properties of the individual nodes and is not improved by increasing $N_{\text {node }}$. Even with an arbitrarily large $N_{\text {node }}$, the limit of detection (LOD) thus still depends on optimizing the node properties, in particular the suppression of false positives $\left(k_{\text {false }}\right.$ $\rightarrow 0)$ and missed events $(\beta \rightarrow 1)$, through both experimental design and signal processing.

Eq 6 indicates that the sensitivity is independent of concentration, but in practice a limit for the maximum measurable concentration will be set by the ability to resolve events that overlap in time at high event rates. This depends largely on the details of the node and is not discussed further here.

\section{IMPLEMENTATION OF DIGITAL SENSING}

A very significant disadvantage of an electrochemical digital sensing architecture is the enormous increase in complexity associated with implementing a (very) large array of separately addressable SEE detectors. Approaches for doing so fall mostly outside the laboratory-based instrumentation typically associated with SEE.

Most high-quality electrodes employed for electrochemistry on the nanoscale rely on macroscopic techniques such as laserassisted pulling. These are eminently unsuited to large-scale parallelization. Material-based methods for creating electrode arrays down to the nanoscale have become increasingly sophisticated, but separately reading out each electrode is challenging (although in principle possible, e.g., by optical methods ${ }^{55}$ ). Simple lithographically based approaches, in which electrodes are patterned on the surface of a chip and passively connected to external instrumentation via individual wires, $^{56}$ are also unfeasible due to the sheer number of connections that would be required between the chip and the outside world.

One way we can begin to address this problem using a purely electrochemical strategy is to employ array architectures for measurements involving two working electrodes. For illustration, consider a generator-collector experiment, in which a species is electrogenerated at one electrode and collected at a second, nearby electrode. The measurement nodes are arranged on a two-dimensional square grid, as illustrated schematically in Figure 3a. Rather than connecting
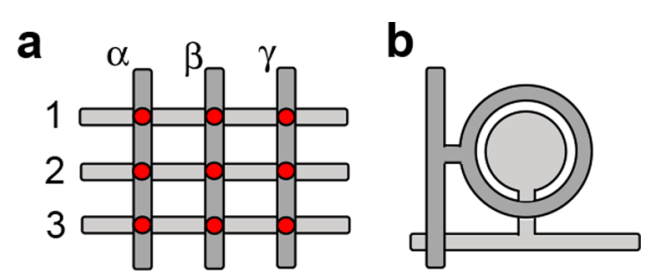

Figure 3. Two-dimensional array architecture. (a) Individual nodes (red dots) can be individually addressed via their row $(1,2,3)$ and column $(\alpha, \beta, \gamma)$ interconnects. In a generator-collector experiment, all of the generator electrodes on the same row are excited simultaneously, and the collector current for each node on that row can be read out via the corresponding column interconnect. Alternatively, in an active array, the row and column wires can control transistor switches to dynamically control which node(s) are active. (b) Example of a generator-collector node geometry in which a disk electrode acts as generator and a surrounding ring electrode acts as collector. The horizontal and vertical bars at the left and bottom represent the row and column interconnects, respectively.

each electrode separately to the outside world, the generator electrode from each node is short-circuited with all the other generator electrodes from the same row, and a single external connection exists for that row. The collector electrodes are similarly connected in a column-wise fashion (Figure $3 \mathrm{~b}$ ). During measurements, a single row is set to a generating potential while the remaining rows and all the columns are set to a collecting potential. In this way, generation takes place only in the row being addressed. The collector current from each node in that row can then be measured by measuring the collector current through the corresponding column, even though each column is also connected to the collector electrodes from the inactive rows. In order to scan the whole array, the active row is cycled in time. For each row, readout of 
the collector current can be done for each column in succession, for several columns at a time, or for all columns in parallel. Such a square array with $N^{2}$ nodes requires $2 N$ wires, a factor $N$ reduction when compared to the $2 N^{2}$ wires needed to connect each electrode separately. This geometry lends itself well to a lithography-based approach in which the individual nodes and their interconnects are fabricated on an insulating substrate.

An important compromise in this approach is that only a subset of the nodes is being actively monitored at any given time. The SEE process under investigation must therefore be sufficiently slow that it can be resolved despite each node being only intermittently probed. Another issue is that the inactive nodes still contribute to the noise and background current of the column signals, degrading the overall noise properties of the measurement.

A more general strategy is to separate the function of addressing individual nodes from that of performing the electrochemical measurement. This requires active switches being incorporated into the array to select which electrode(s) are polarized and/or read out. This approach lends itself naturally to an integrated circuit implementation in which the active elements take the form of transistors that are incorporated together with the electrodes and their interconnects on the same chip. Having taken that step, it becomes possible to implement additional functionality on the chip such as amplification and even digitization.

In recent decades, a wide variety of electrochemical instrumentation has been implemented using complementary metal-oxide-semiconductor (CMOS) technology, the workhorse of the semiconductors industry. While this field is far too broad to review here, ${ }^{57}$ some recent examples of CMOS-based massively parallelized electrochemical devices include ionsensitive field-effect transistors (ISFETs) for DNA sequencing, ${ }^{58}$ microelectrode arrays (MEAs) for electrogenic cell recording and stimulation, ${ }^{59,60}$ and high-density nanocapacitor arrays for high-frequency detection. ${ }^{81}$ This directly exploits some of the strengths of CMOS technology, in particular the means to create miniaturized electrode structures (although this can require postprocessing following standard CMOS fabrication), the ability to arrange these in large arrays of separately addressable nodes, high switching speeds, and the flexibility to integrate additional functionality.

Implementing electrochemical instrumentation on a chip may however entail significant compromises. For example, $1 / f$ noise plays an increasingly large role upon decreasing transistor dimensions. $^{62}$ This directly impacts the noise properties of transimpedance amplifiers employed in amperometry (the method of choice for many SEE experiments, as illustrated in Figure 1 ). While $1 / f$ noise is not a main limiting factor in many present-day implementations, ${ }^{63}$ it can severely limit downscaling amperometric arrays to the submicron scale. This may ultimately favor transducers that are less sensitive to $1 / f$ noise, such as high-frequency switched capacitor methods. ${ }^{64,65}$

\section{SUMMARY AND OUTLOOK}

SEE is a rapidly evolving subfield that is extending fundamental understanding and pushing the capabilities of electrochemical techniques into new territory. While SEE detectors have exquisite mass sensitivity, translating this into a high concentration sensitivity still represents a formidable challenge. This is in no small part due to the size mismatch between typical SEE detectors and the much larger dimensions that are required to overcome the limitations imposed by mass transport and binding kinetics. Large-scale parallelization suggests a solution to this conundrum, but this comes at a significant increase in complexity and most likely requires implementing digital SEE sensing in the form of integrated circuits. This necessarily involves significant design compromises since microelectronic processing and circuit architectures are not always in harmony with electrode materials and instrumentation most commonly employed in electrochemistry.

A potentially disruptive factor that we have ignored in our analysis of mass transport and binding kinetics is targeted active transport. There is a growing set of methods in microand nanofluidics for selectively enriching the local concentration of target molecules at a sensor (e.g., isotachophoresis, ${ }^{66}$ ion concentration polarization focusing, ${ }^{67} \mathrm{AC}$ electrokinetic methods $\left.{ }^{68}\right)$. These techniques are beneficial for both conventional and digital sensors, but in particular they may help alleviate the stringent requirements for implementing digital detection by decreasing the minimum number of nodes.

A key ingredient of (bio)sensors that has not yet been explicitly addressed at the experimental level for most SEE methods is receptor compatibility. So far, many SEE measurements have focused on the development of signal transduction methods with minimal attention to specificity. How best to couple an affinity-based assay to most SEE detection methods remains a largely open question. This is further complicated by the fact that electrochemical processes can locally influence biochemical kinetics, an encouraging example being the enhancement of the rate of DNA hybridization at an electrode under potential modulation. ${ }^{69}$

Can SEE-based digital (bio)sensors advance to the point where they can revolutionize trace-level detection? This is at the moment an open question. Most SEE methods are still at an early stage of development, and the technical challenges ahead are very significant. By comparison, optical methods provide a more straightforward entry for the development of digital sensing concepts. This is in no small part due to digital cameras that permit high-sensitivity measurements over large areas and with high spatial resolution. The SEE equivalent of mature CCD and CMOS image sensors does not exist yet. It is difficult to underestimate the potential of CMOS technology, however. Past the initial hurdle of development, in terms of both time and resources, all-electrical assays incorporating colossal numbers of active elements could be mass produced at low cost. For this to happen, however, will require continued close collaboration between nanoelectrochemists and circuit engineers.

\section{ASSOCIATED CONTENT}

Supporting Information

The Supporting Information is available free of charge at https://pubs.acs.org/doi/10.1021/acs.analchem.1c00510.

Derivation of eq 5 (PDF)

\section{AUTHOR INFORMATION}

\section{Corresponding Author}

Serge G. Lemay - MESA+ Institute for Nanotechnology and Faculty of Science and Technology, University of Twente, 7500 AE Enschede, The Netherlands; (1) orcid.org/00000002-0404-3169; Email: s.g.lemay@utwente.nl 


\section{Author}

Taghi Moazzenzade - MESA+ Institute for Nanotechnology and Faculty of Science and Technology, University of Twente, 7500 AE Enschede, The Netherlands; 10 orcid.org/00000002-3908-6062

Complete contact information is available at:

https://pubs.acs.org/10.1021/acs.analchem.1c00510

\section{Notes}

The authors declare no competing financial interest.

\section{ACKNOWLEDGMENTS}

We thank F. Widdershoven and L. Selmi for critical comments on the manuscript, the SENTINEL network for useful discussions (funded by the European Union's Horizon 2020 Research and Innovation Program under Marie SkłodowskaCurie grant agreement no. 812398), and The Netherlands TopSector High-Tech Systems \& Materials for financial support under the TKI project "Early Cancer Diagnostics".

\section{REFERENCES}

(1) Baker, L. A. J. Am. Chem. Soc. 2018, 140, 15549-15559.

(2) Coulter, W. H. U.S. Patent US2656508, 1953.

(3) Li, J.; Stein, D.; McMullan, C.; Branton, D.; Aziz, M. J.; Golovchenko, J. A. Nature 2001, 412, 166-169.

(4) Li, J.; Gershow, M.; Stein, D.; Brandin, E.; Golovchenko, J. A. Nat. Mater. 2003, 2, 611-615.

(5) Kasianowicz, J. J.; Brandin, E.; Branton, D.; Deamer, D. W. Proc. Natl. Acad. Sci. U. S. A. 1996, 93, 13770-13773.

(6) Clarke, J.; Wu, H.-C.; Jayasinghe, L.; Patel, A.; Reid, S.; Bayley, H. Nat. Nanotechnol. 2009, 4, 265-270.

(7) Deamer, D.; Akeson, M.; Branton, D. Nat. Biotechnol. 2016, 34, 518-524.

(8) Jain, M.; Koren, S.; Miga, K. H.; Quick, J.; Rand, A. C.; Sasani, T. A.; Tyson, J. R.; Beggs, A. D.; Dilthey, A. T.; Fiddes, I. T.; et al. Nat. Biotechnol. 2018, 36, 338-345.

(9) Depledge, D. P.; Srinivas, K. P.; Sadaoka, T.; Bready, D.; Mori, Y.; Placantonakis, D. G.; Mohr, I.; Wilson, A. C. Nat. Commun. 2019, 10,754 .

(10) Liu, Y.; Li, M.; Zhang, F.; Zhu, A.; Shi, G. Anal. Chem. 2015, $87,5531-5538$.

(11) Luo, L.; White, H. S. Langmuir 2013, 29, 11169-11175.

(12) Neher, E.; Sakmann, B. Nature 1976, 260, 799-802.

(13) Hamill, O. P.; Marty, A.; Neher, E.; Sakmann, B.; Sigworth, F. J. Pfluegers Arch. 1981, 391, 85-100.

(14) Liu, H. Y.; Fan, F. R. F.; Lin, C. W.; Bard, A. J. J. Am. Chem. Soc. 1986, 108, 3838-3839.

(15) Itaya, K.; Tomita, E. Surf. Sci. 1988, 201, L507-L512.

(16) Ebejer, N.; Schnippering, M.; Colburn, A. W.; Edwards, M. A.; Unwin, P. R. Anal. Chem. 2010, 82, 9141-9145.

(17) Kleijn, S. E. F.; Lai, S. C. S.; Miller, T. S.; Yanson, A. I.; Koper, M. T. M.; Unwin, P. R. J. Am. Chem. Soc. 2012, 134, 18558-18561.

(18) Bentley, C. L.; Kang, M.; Unwin, P. R. Current Opinion in Electrochemistry 2017, 6, 23-30.

(19) Fan, F. R. F.; Bard, A. J. Science 1995, 267, 871-874.

(20) Zevenbergen, M. A. G.; Singh, P. S.; Goluch, E. D.; Wolfrum, B. L.; Lemay, S. G. Nano Lett. 2011, 11, 2881-2886.

(21) Quinn, B. M.; van't Hof, P. G.; Lemay, S. G. J. Am. Chem. Soc. 2004, 126, 8360-8361.

(22) Xiao, X.; Bard, A. J. J. Am. Chem. Soc. 2007, 129, 9610-9612.

(23) Zhou, Y. G.; Rees, N. V.; Compton, R. G. Angew. Chem., Int. Ed. 2011, 50, 4219-4221.

(24) Banks, C. E.; Rees, N. V.; Compton, R. G. J. Phys. Chem. B 2002, 106, 5810-5813.

(25) Visser, E. W. A.; Yan, J.; van Ijzendoorn, L. J.; Prins, M. W. J. Nat. Commun. 2018, 9, 2541.
(26) Nouri, R.; Tang, Z.; Guan, W. Anal. Chem. 2019, 91, 1117811184.

(27) Jing, W.; Wang, Y.; Yang, Y.; Wang, Y.; Ma, G.; Wang, S.; Tao, N. ACS Nano 2019, 13, 8609-8617.

(28) Huang, Q.; Li, N.; Zhang, H.; Che, C.; Sun, F.; Xiong, Y.; Canady, T. D.; Cunningham, B. T. Lab Chip 2020, 20, 2816-2840.

(29) Teh, S.-Y.; Lin, R.; Hung, L.-H.; Lee, A. P. Lab Chip 2008, 8, 198-220.

(30) Guo, M. T.; Rotem, A.; Heyman, J. A.; Weitz, D. A. Lab Chip 2012, 12, 2146-2155.

(31) Rissin, D. M.; Kan, C. W.; Campbell, T. G.; Howes, S. C.; Fournier, D. R.; Song, L.; Piech, T.; Patel, P. P.; Chang, L.; Rivnak, A. J.; et al. Nat. Biotechnol. 2010, 28, 595-599.

(32) Cohen, L.; Walt, D. R. Annu. Rev. Anal. Chem. 2017, 10, 345363.

(33) Kwon, S. J.; Fan, F.-R. F.; Bard, A. J. J. Am. Chem. Soc. 2010, 132, 13165-13167.

(34) Lebègue, E.; Anderson, C. M.; Dick, J. E.; Webb, L. J.; Bard, A. J. Langmuir 2015, 31, 11734-11739.

(35) Li, X.; Batchelor-McAuley, C.; Compton, R. G. ACS Sensors 2019, 4, 464-470.

(36) Chen, C.-H.; Ravenhill, E. R.; Momotenko, D.; Kim, Y.-R.; Lai, S. C. S.; Unwin, P. R. Langmuir 2015, 31, 11932-11942.

(37) Shi, X.; Verschueren, D. V.; Dekker, C. Nano Lett. 2018, 18, 8003-8010.

(38) Moazzenzade, T.; Yang, X.; Walterbos, L.; Huskens, J.; Renault, C.; Lemay, S. G. J. Am. Chem. Soc. 2020, 142, 17908-17912.

(39) Sheehan, P. E.; Whitman, L. J. Nano Lett. 2005, 5, 803-807.

(40) Liu, X.; Lu, J.; Cha, C. J. Electroanal. Chem. Interfacial Electrochem. 1990, 294, 289-292.

(41) Oldham, K. B. J. Electroanal. Chem. 1992, 323, 53-76.

(42) Bard, A. J.; Faulkner, L. R. Electrochemical Methods: Fundamentals and Applications; John Wiley \& Sons: New York, 2001.

(43) Langmuir, I. J. Am. Chem. Soc. 1918, 40, 1361-1403.

(44) Liebermann, T.; Knoll, W.; Sluka, P.; Herrmann, R. Colloids Surf., A 2000, 169, 337-350.

(45) Squires, T. M.; Messinger, R. J.; Manalis, S. R. Nat. Biotechnol. 2008, 26, 417.

(46) Morf, W. E. Anal. Chim. Acta 1996, 330, 139-149.

(47) Sokolov, S. V.; Bartlett, T. R.; Fair, P.; Fletcher, S.; Compton, R. G. Anal. Chem. 2016, 88, 8908-8912.

(48) Bilmes, J. Techanical Report; University of California: Berkeley, 1998.

(49) Eddy, S. R. Nat. Biotechnol. 2004, 22, 1315-1316.

(50) Chung, S. H.; Moore, J. B.; Xia, L.; Premkumar, L. S.; Gage, P. W. Philos. Trans. R. Soc., B 1990, 329, 265-285.

(51) McKinney, S. A.; Joo, C.; Ha, T. Biophys. J. 2006, 91, 19411951.

(52) Bronson, J. E.; Fei, J. Y.; Hofman, J. M.; Gonzalez, R. L.; Wiggins, C. H. Biophys. J. 2009, 97, 3196-3205.

(53) Kostiuchenko, Z. A.; Zhang, B.; Lemay, S. G. J. Phys. Chem. C 2016, 120, 22777-22783.

(54) Ekins, R.; Edwards, P. Clin. Chem. 1997, 43, 1824-1831.

(55) Fan, Y.; Anderson, T. J.; Zhang, B. Current Opinion in Electrochemistry 2018, 7, 81-86.

(56) Rassaei, L.; Singh, P. S.; Lemay, S. G. Anal. Chem. 2011, 83, 3974-3980.

(57) Li, H. T.; Liu, X. W.; Li, L.; Mu, X. Y.; Genov, R.; Mason, A. J. Sensors 2017, 17, 74.

(58) Rothberg, J. M.; Hinz, W.; Rearick, T. M.; Schultz, J.; Mileski, W.; Davey, M.; Leamon, J. H.; Johnson, K.; Milgrew, M. J.; Edwards, M.; et al. Nature 2011, 475, 348-352.

(59) Ballini, M.; Muller, J.; Livi, P.; Yihui Chen; Frey, U.; Stettler, A.; Shadmani, A.; Viswam, V.; Lloyd Jones, I.; Jackel, D.; Radivojevic, M.; Lewandowska, M. K.; Wei Gong; Fiscella, M.; Bakkum, D. J.; Heer, F.; Hierlemann, A.; et al. IEEE J. Solid-State Circuits 2014, 49, 2705-2719.

(60) Tsai, D.; Sawyer, D.; Bradd, A.; Yuste, R.; Shepard, K. L. Nat. Commun. 2017, 8, 1802. 
(61) Laborde, C.; Pittino, F.; Verhoeven, H. A.; Lemay, S. G.; Selmi, L.; Jongsma, M. A.; Widdershoven, F. P. Nat. Nanotechnol. 2015, 10, 791-795.

(62) Tuinhout, H.; Duijnhoven, A. Z. In Evaluation of $1 / F$ Noise Variability in the Subthreshold Region of Mosfets, 2013 IEEE International Conference on Microelectronic Test Structures (ICMTS), March 25-28, 2013; pp 87-92.

(63) Hartel, A. J. W.; Shekar, S.; Ong, P.; Schroeder, I.; Thiel, G.; Shepard, K. L. Anal. Chim. Acta 2019, 1061, 13-27.

(64) Widdershoven, F.; Cossettini, A.; Laborde, C.; Bandiziol, A.; van Swinderen, P. P.; Lemay, S. G.; Selmi, L. IEEE Transactions on Biomedical Circuits and Systems 2018, 12, 1369-1382.

(65) Ieee. 2019 Ieee Biomedical Circuits and Systems Conference 2019, 1.

(66) Smejkal, P.; Bottenus, D.; Breadmore, M. C.; Guijt, R. M.; Ivory, C. F.; Foret, F.; Macka, M. Electrophoresis 2013, 34, 14931509.

(67) Berzina, B.; Anand, R. K. Anal. Chim. Acta 2020, 1128, 149173.

(68) Salari, A.; Thompson, M. Sens. Actuators, B 2018, 255, 36013615.

(69) Tymoczko, J.; Schuhmann, W.; Gebala, M. ACS Appl. Mater. Interfaces 2014, 6, 21851-21858. 